\title{
Rapid and Reliable Measurement of Highly Elevated Blood Ammonia Concentrations in Children
}

\author{
Marinus H. de Keijzer ${ }^{1}$, Bernadette S. Jakobs ${ }^{1}$, Rob W. Brandts ${ }^{1}$, Marc T. W. Hofs ${ }^{1}$, Frans J. M. Trijbels ${ }^{2}$ and \\ Jan A. M. Smeitink ${ }^{2}$ \\ 1 Department of Clinical Chemistry \\ 2 Department of Metabolic Diseases \\ University Hospital St. Radboud, Nijmegen, The Netherlands
}

Summary: Newborns and children may suffer from extremely high ammonia levels in their blood. We evaluated a fast, reliable micromethod, based on the Blood Ammonia Checker II (BAC II) in combination with the dilution with fresh whole blood. Comparison of the proposed method with an enzymatic method revealed a statistically significant correlation. We conclude that the dilution of patient's blood with fresh whole blood extends the measuring range of ammonia on the BAC II analyzer from $286 \mu \mathrm{mol} / 1$ to about $700 \mu \mathrm{mol} / 1$.

\section{Introduction}

Within one month two neonates and one infant with extremely high levels of ammonia in blood were admitted to our hospital. They were suffering from three different inborn errors of metabolism: argininosuccinate lyase deficiency, pyruvate carboxylase deficiency and carnitinepalmitoyltransferase type II deficiency. Blood ammonia concentrations in all three comatous children varied between 450 and $800 \mu \mathrm{mol} / 1$ (normal concentration: less than $60 \mu \mathrm{mol} / 1)$. Continuous arterio-venous haemofiltration in the two neonates and repeated blood exchange in the infant were performed, in combination with intravenously given high-dose glucose and disease-specific drugs in order to normalize the ammonia levels as soon as possible.

Ammonia derived from the deamination of amino acids is detoxificated via conversion to glutamine, which is subsequently transported to the liver. In the liver, glutamine and glutamate are deaminated, forming ammonia, which in turn is efficiently converted to urea. The waste product urea is then excreted into the urine. In patients with liver failure or various inborn errors of metabolism, very high levels of ammonia may be present. As paediatricians need these levels rapidly to monitor the efficiency of ammonia detoxification, we developed and evaluated a fast and reliable micro-method for the measurement of extremely elevated ammonia levels in children.

\section{Materials and Methods}

Experimental design

In our laboratory we use two different methods to determine the concentration of ammonia in blood. The first one is the Blood Am- monia Checker II (BAC II) (Kyoto Daiichi Kaguku Co., Ltd. Japan) which needs $20 \mu \mathrm{l}$ whole blood for each determination (1). Measurements are performed in duplicate with a difference of less than $10 \%$ in the normal range and less than $5 \%$ in the pathological range. The analyzer is a reflectance meter using special reagent strips with bromocresol. Results are available within 3 minutes; this and the small sample volume makes the BAC II very suitable for STAT investigations and for paediatric samples. However, in case of extremely high levels of ammonia this method is insufficient since the upper limit of ammonia concentration is only 286 $\mu \mathrm{mol} / \mathrm{l}$. Therefore, we also use the Monotest kit (Boehringer Mannheim, Almere, The Netherlands) in combination with the Cobas Mira Plus (Roche Diagnostic Systems, Mijdrecht, The Netherlands). This enzymatic assay is based on the conversion of 2-oxoglutarate and ammonia to glutamate with glutamate dehydrogenase (2). The measurements are performed in plasma with a coefficient of variation $(\mathrm{CV})$ of less than $3 \%$ and the whole procedure takes more than one hour. This makes this method unsuitable for the investigation of acute metabolic deteriorations.

To evaluate a modification of the BAC II method we increased the ammonia concentration in whole blood samples artificially, diluted these samples with fresh whole blood (with low ammonia concentration), measured the samples on the BAC II analyzer and compared the results with those measured by the enzymatic method.

Fifteen fresh EDTA-anticoagulated blood samples $(1 \mathrm{ml})$ were spiked with $2.5 \mu \mathrm{l}, 6.5 \mu \mathrm{l}$ or $9.0 \mu \mathrm{l}$ of a solution containing ammonium sulphate with a concentration of $80 \mathrm{mmol} / \mathrm{l}$. After 10 minutes half of the sample was transferred to another tube, centrifuged and the plasma was placed on ice until ammonia measurements on the Cobas analyzer were carried out. The other half of the sample was diluted with fresh EDTA-anticoagulated blood from outward patients. Dilutions were 1:1 to 1:5, depending on the volume of ammonium sulphate added. Immediately after mixing ammonia levels were measured in duplicate with the BAC II analyser.

The Cobas samples were analysed in triplicate. The analyzer dilutes the sample with distilled water when the concentration of ammonia is above $250 \mu \mathrm{mol} / \mathrm{l}$. Above $500 \mu \mathrm{mol} / /$ a predilution with saline is necessary. Ammonia Preciset standards (Boehringer Mannheim, Almere, The Netherlands) were used for calibration.

Linear regression analysis was performed with the non-parametric method of Passing \& Bablok (3). 


\section{Results}

The ammonia concentrations of the fresh blood samples varied from 24 to $59 \mu \mathrm{mol} / \mathrm{l}$. These amounts were used to calculate the ammonia concentration of the spiked blood samples. Calculation was done with the formula:

$$
\text { conc }=(A-F) x+F
$$

in which $\mathrm{A}$ is the ammonia concentration of the diluted sample, $\mathrm{F}$ is the ammonia concentration of the fresh blood sample and $\mathrm{x}$ is the dilution factor. From the results of the duplicate measurements on the BAC II analyzer CVs of $1.8 \%, 1.7 \%$ and $2.8 \%$ were calculated for the ranges between 300 to $500 \mu \mathrm{mol} / 1,500$ to $700 \mu \mathrm{mol} / 1$ and 700 to $900 \mu \mathrm{mol} / 1$. The triplicate measurements with the Cobas Mira Plus analyzer resulted in CVs of $1.1 \%$, $1.3 \%$ and $0.8 \%$, respectively.

The results of the BAC II analyzer versus the automated method are depicted in figure 1 . Linear regression analysis revealed a line with the equation $\mathrm{y}=1.1 \mathrm{x}-19.1$ $(r=0.96)$. From the figure it can be concluded that the proposed method measures blood ammonia concentrations up to a level of $700 \mu \mathrm{mol} / \mathrm{l}$ reliably.

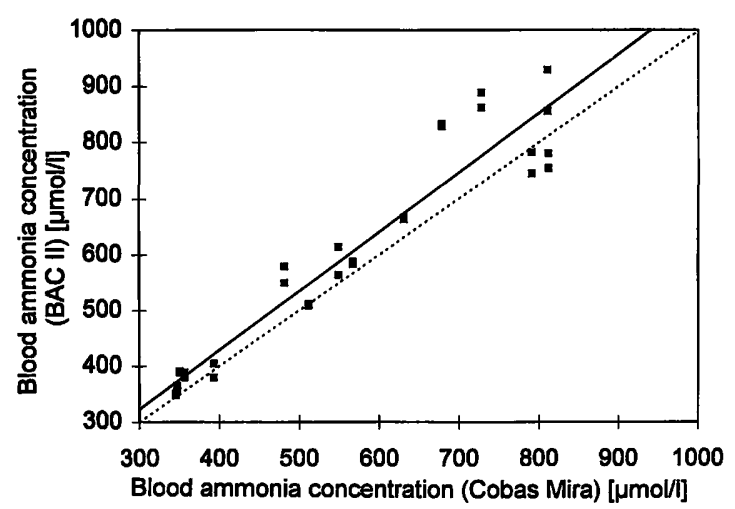

Fig. 1 Blood ammonia concentrations determined on the BAC II analyzer after dilution with fresh whole blood and compared with results obtained with an enzymatic Cobas method.

$\mathrm{y}=1.1 \mathrm{x}-19.1 ; \mathrm{r}=0.96$

The dotted line represents complete identity $(y=x)$.

\section{References}

1. Huizenga JR, Tangerman A, Gips $\mathrm{CH}$. A rapid method for blood ammonia determination using the new Blood Ammonia Checker (BAC) II. Clin Chim Acta 1992; 210:153-5.

2. Da Fonseca-Wolheim F. Direkte Plasmaammoniakbestimmung ohne Enteiweissung. J Clin Chem Clin Biochem 1973; 11:421-31.

3. Passing $\mathrm{H}$, Bablok W. Comparison of several regression procedures for method comparison studies and determination of sample size. Part II. J Clin Chem Clin Biochem 1984; 22:431-5.

4. Walter JH, Leonard JV. Inborn errors of the urea cycle. Br J Hosp Med 1987; 38:176-83.

\section{Discussion}

Children with certain inherited metabolic diseases may suffer from extremely elevated levels of ammonia ( $>500 \mu \mathrm{mol} / 1)$. Hyperammonaemic crisis in newborns and children is a real emergency situation. The time spent in hyperammonaemic coma is critical because there is a significant correlation between the level of ammonia and the duration of the coma and the outcome or prognosis $(4,5)$. Therefore, fast and frequent ammonia measurements, immediate treatment and continuous monitoring of the child has to be performed as soon as possible. The BAC II analyzer offers the opportunity to monitor the efficiency of ammonia detoxification in a very easy and rapid way, which makes the apparatus especially valuable in these cases. The upper limit, however, is below $286 \mu \mathrm{mol} / 1$, which is insufficient in a number of these children. In an attempt to extend the measuring range, we diluted blood samples (with high ammonia concentrations due to the addition of ammonium sulphate) with fresh whole blood and measured the blood ammonia concentration on the BAC II analyzer. To evaluate this fresh blood dilution method we compared the results from the BAC II with those obtained with an enzymatic method. Dilution was performed with fresh whole blood since saline or other aqueous solutions caused discrepancies between the results. Several reasons may explain this phenomenon: a difference in viscosity will cause a different diffusion rate after the application of the sample on the strip. Moreover, the decrease of the haematocrit and/or of the concentration of albumin will also lead to unreliable results (6).

Since overall precision was identical with both methods and fresh whole blood dilutions extended the measuring range of the BAC II analyzer up to ammonia concentrations of $700-800 \mu \mathrm{mol} / \mathrm{l}$, we conclude that this method measures - very fast and reliable - extremely elevated ammonia levels in blood.

\section{Acknowledgements}

We are indebted to Mr. S. M. Klaver for his expert technical assistance.

5. Msall M, Batshaw ML, Suss R, Brusilow SW, Mellits ED. Neurologic outcome in children with inborn errors of urea synthesis. Outcome of urea-cycle enzymopathies. N Engl J Med $1984 ; 310: 1500-5$.

6. Manual Blood Ammonia Checker II, Menarini Diagnostics, Valkenswaard, The Netherlands.

Received June 27/ September 4, 1997

Corresponding author: Dr. M H. de Keijzer, PhD, University

Hospital St Radboud, 564 CKCL, PO Box 9101,

NL-6500 HB Nijmegen, The Netherlands 